\title{
A triple action CDK4/6-PI3K-BET inhibitor with augmented cancer cell cytotoxicity
}

\author{
Adam M. Burgoyne ${ }^{1}$, Kendra R. Vann², Shweta Joshi ${ }^{3}$, Guillermo A. Morales ${ }^{4}$, Francisco M. Vega $\mathbb{C}^{3,5}$, Alok Singh ${ }^{3}$, \\ Dhananjaya Pal ${ }^{3}$, Aran B. Merati ${ }^{1}$, Tatiana G. Kutateladze $\mathbb{C}^{2}$ and Donald L. Durden ${ }^{3,4}$
}

Dear Editor,

The PI3K-AKT-mTOR pathway has been at the center of anti-cancer drug development ${ }^{1,2}$, but targeted inhibition of PI3K kinase activity is proven to provide a limited therapeutic effect and is often followed by the development of resistance to the drug $^{3}$. This limitation could in part be alleviated through concurrently inhibiting multiple pathological pathways characterized by synthetic lethality relationship. For example, beneficial effects in metastatic breast cancer are observed when inhibition of PI3K is combined with BET proteins inhibition ${ }^{4}$. While the need for combinations of single agent targeted therapies has become clear, this approach commonly requires dose reduction of the individual agents due to additive toxicity that may compromise efficacy ${ }^{5}$.

One strategy being explored to overcome the drawbacks of combination-based targeted therapy relies on the development of single-molecule inhibitors highly specific toward several targets. We and others have previously reported potent dual activity inhibitors, including PI3KBET inhibitors ${ }^{6,7}$, cyclin-dependent kinases (CDK)-BET and mitogen-activated protein kinases (MAPK)-BET inhibitors ${ }^{8}$, and CDK-PI3K inhibitors ${ }^{9}$. In this study, we describe the first in class rationally-designed triple activity inhibitor that concomitantly disrupts functions of three critical targets based upon known synthetic lethality relationships in cancer cells-CDK4/6, PI3K, and BRD4. The triple inhibitor SRX3177 has broad cytotoxic activity against a variety of tumor types. Our results using a cyclin

\footnotetext{
Correspondence: Tatiana G. Kutateladze (tatiana.kutateladze@cuanschutz.edu) or Donald L. Durden (ddurden@ucsd.edu)

${ }^{1}$ Division of Hematology-Oncology, Department of Medicine, Moores Cancer Center, University of California San Diego, La Jolla, CA, USA

${ }^{2}$ Department of Pharmacology, University of Colorado School of Medicine,

Aurora, CO, USA

Full list of author information is available at the end of the article

These authors contributed equally: Adam M. Burgoyne, Kendra R. Vann
}

D1-dependent hematologic malignancy, a MYCdependent embryonal tumor, and a PI3K-dependent solid tumor demonstrate that SRX3177 is efficacious and non-toxic in vitro to normal epithelial cells.

We originally generated a series of chemical probes that simultaneously bind CDK4/6, PI3K, and BRD4 based on analyses of the crystal structures of the target domains and in silico modeling. By screening these compounds against the three targets using in vitro binding assays, kinase assays, and CDK assays, we identified the most potent compound, SRX3177 (Fig. 1a). SRX3177 showed nanomolar potency against PI3Ka, both bromodomains of BRD4 (BD1 and BD2), and CDK4/6 (Fig. 1b). The specificity of SRX3177 and lack of off-targeting was confirmed by KINOMEscan and BROMOscan assays (Fig. 1c, d and Supplementary Table S1).

To gain insight into the molecular basis for inhibition of BRD4, we assessed binding of SRX3177 to BD1 and BD2 of BRD4 using NMR and X-ray crystallographic approaches. Large chemical shift perturbations in ${ }^{1} \mathrm{H},{ }^{15} \mathrm{~N}$ heteronuclear single quantum coherence (HSQC) spectra of ${ }^{15} \mathrm{~N}$-labeled BRD4 BD1 and BD2 were observed upon gradual addition of SRX3177, demonstrating that SRX3177 directly targets both bromodomains of BRD4 (Supplementary Fig. S1). A slow-to-intermediate exchange regime on the NMR chemical shift time scale indicated a tight interaction, in full agreement with the nanomolar values of $\mathrm{K}_{\mathrm{d}}$ and $\mathrm{IC}_{50}$.

To obtain the atomic-resolution mechanistic details, we co-crystallized BRD4 BD1 with SRX3177 and determined the crystal structure of the BD1-SRX3177 complex (Supplementary Table S2). In the complex, SRX3177 occupies a large hydrophobic pocket located at one of the open ends of the BD1 four-helix bundle (Fig. 1e and Supplementary Fig. S2a, b). The base of the SRX3177binding pocket is lined with a well-defined water shell, 


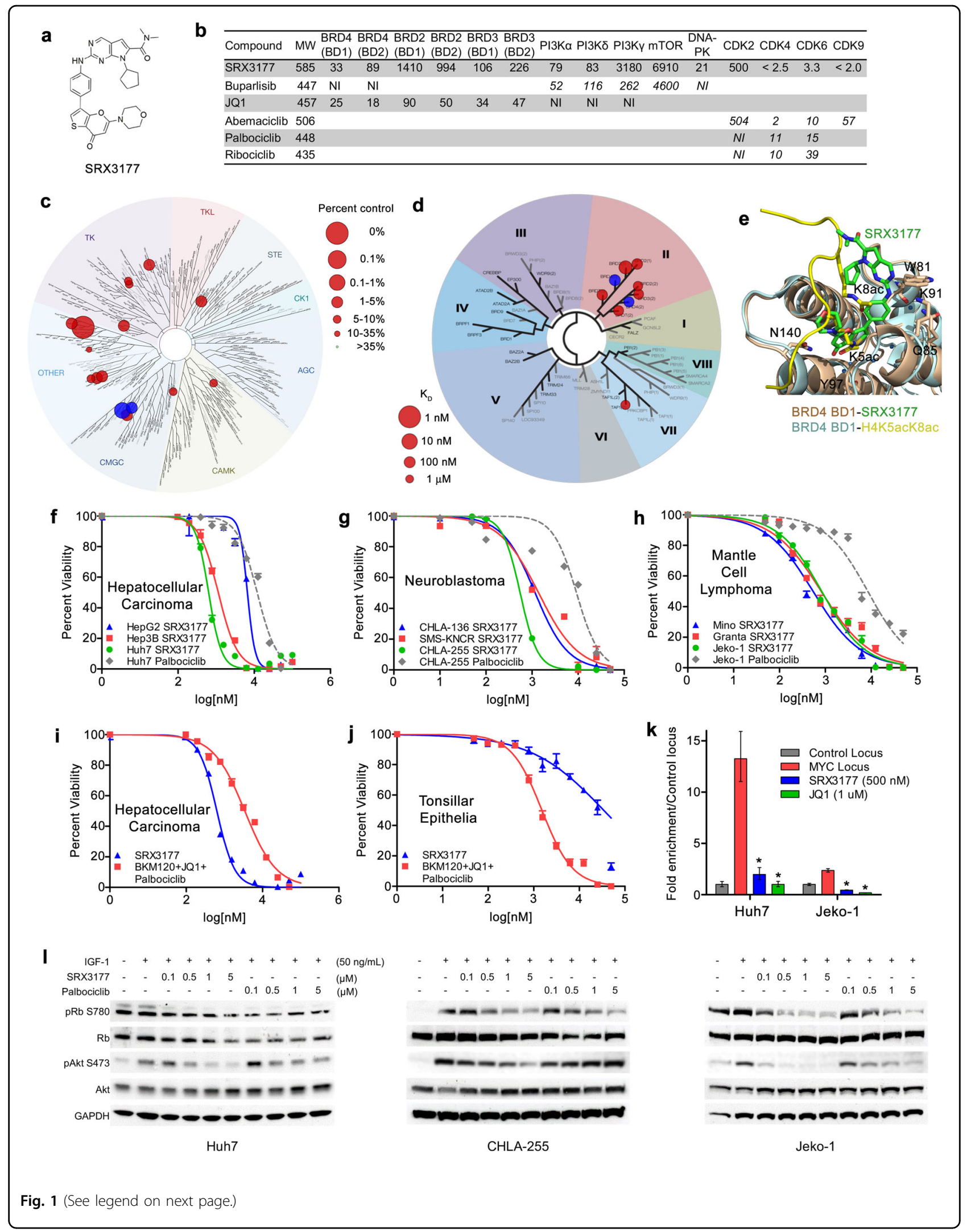


(see figure on previous page)

Fig. 1 SRX3177 is a novel triple action inhibitor. a Structure of SRX3177. $\mathbf{b}$ In vitro bromodomain binding and kinase activities were performed to measure $\mathrm{IC}_{50}$ values which are displayed as $\mathrm{nM}$ concentrations. Italicized values were obtained from the literature. $\mathbf{c}$, $\mathbf{d}$ KINOMEscan (c) and BROMOscan (d) data were analyzed using the TREEspot. Target effects are indicated by red circles. e Structural basis for the recognition of SRX3177 by BRD4 BD1. Overlay of the structures of BRD4 BD1 (light brown) in complex with SRX3177 (green) and H4K5acK8ac peptide (PDB: 3UVW) (yellow). f-h Hepatocellular carcinoma (HepG3, Hep3B, and Huh7), neuroblastoma (CHLA-136, SMS-KNCR, and CHLA-255), and mantle cell lymphoma (Mino, Granta-519, and Jeko-1) were treated with increasing doses of SRX3177 or palbociclib and assessed for viability for determination of IC $50 . \mathbf{i}, \mathbf{j}$ Huh7 and normal tonsillar epithelial cells were treated with SRX3177 versus combination of BKM120, JQ1, and palbociclib and assessed for viability and IC 50 . k ChIP assays using Huh7 and Jeko-1 cells treated with SRX3177. Purified immunoprecipitated DNA and input DNA was amplified by quantitative real-time PCR and analyzed for enriched binding of BRD4 to the MYC transcription start site in comparison with binding to a nonspecific locus upstream of MYC. Data were normalized and plotted as percentage of vehicle control binding. Asterisks denote $p \leq 0.05$ in comparison with vehicle control by unpaired Student's t-test. I Serum starved Huh7, CHLA-255, and Jeko-1 cells were stimulated with $50 \mathrm{ng} / \mathrm{mL}$ IGF-1 and treated with SRX3177 or palbociclib. Lysates were immunoblotted for pRb, Rb, pAkt, Akt, and GAPDH.

which is conserved in other BRD4 BD1 complexes ${ }^{10}$. Superimposition of the SRX3177-bound and H4K5acK8ac-bound BRD4 BD1 structures reveals that SRX3177 acts as an acetyllysine mimetic. Particularly, a hallmark contact, which is required for functional bromodomains, involves formation of the hydrogen bond between the acetyllysine substrate and the side chain of Asn140. In the BD1-SRX3177 complex, the side chain of Asn140 is hydrogen bonded to the thienopyrano group of SRX3177 (Supplementary Fig. S2c).

We then tested the efficacy of SRX3177 in the NCI-60 Panel of Human Tumor Cell Line Screen at the National Cancer Institute, in which the triple inhibitor SRX3177 had cytotoxic effects on the majority of the cell lines tested, with activity against cells derived from all underlying tumor histologies (Supplementary Fig. S3). SRX3177 displayed greater tumor cytotoxicity as compared to the dual BRD4/PI3K inhibitor, SF2523 or dual BRD4/CDK4/6 inhibitor, SRX3177P (Supplementary Fig. S3). To characterize the effect of SRX3177 in detail, we measured cytotoxic activity of this inhibitor in a cyclin D1-dependent hematologic malignancy (mantle cell lymphoma), a MYCdependent embryonal tumor (neuroblastoma), and a PI3K-dependent solid tumor (hepatocellular carcinoma) (Fig. 1f-h). The dose-response curves generated from cellbased cytotoxicity assays in panels of cell lines from each of these tumor types revealed a strong antitumor activity of SRX3177, with $\mathrm{IC}_{50}$ values in the nanomolar range (Fig. 1f-h and Supplementary Table S3). Furthermore, SRX3177 was $\sim 10-20$-fold more potent than the CDK4/6 inhibitor palbociclib in these tumor cell lines.

To further evaluate the anticancer activity of SRX3177, we compared the cytotoxic effects caused by SRX3177 to the cytotoxic effects caused by a combination of individual PI3K, BRD4, and CDK4/6 inhibitors of similar potency (Fig. 1i, j). Huh7 cells were treated with either SRX3177 or the combination of BKM120 (buparlisib), JQ1, and palbociclib and assessed for cytotoxicity. Equimolar combination of the three drugs had a combined $\mathrm{IC}_{50}$ of $3.4 \mu \mathrm{M}$, which is 5 -fold less potent than that of SRX3177 (Fig. 1i). Furthermore, when normal tonsillar epithelial cells were treated with either SRX3177 or the combination of BKM120, JQ1, and palbociclib (as a surrogate for toxicity of normal tissue), the toxicity was increased 20-fold (Fig. 1j). Altogether, these data suggest that the triple inhibitor SRX3177 has higher efficacy and is substantially less toxic to normal cells in vitro in comparison with the combination of individual CDK4/6, PI3K and BRD4 inhibitors of similar potency.

To examine the effect of SRX3177 on BRD4 chromatin binding function, we measured occupancy of BRD4 at the transcriptional start site (TSS) of $M Y C$, a known target of BRD4, by chromatin immunoprecipitation (ChIP). Treatment of Huh7 and Jeko-1 cells with SRX3177 abrogated binding of BRD4 to the MYC TSS, indicating that SRX3177 has pharmacodynamic activity against its BRD4 target in cell-based models (Fig. 1k). Finally, immunoblotting of lysates from Huh7, CHLA-255, and Jeko-1 cells stimulated with IGF-1 and treated with increasing concentrations of SRX3177 showed a decrease in levels of Rb phosphorylation (as a downstream surrogate of CDK4/6 activity) and Akt phosphorylation (as a downstream surrogate of PI3K activity) (Fig. 1l). Thus, SRX3177 effectively inhibits its targets BRD4, CDK4/6, and PI3K in cell-based assays.

In conclusion, in this study we report the first triple action single-molecule inhibitor SRX3177, which disrupts cancer cell signaling through simultaneously inhibiting CDK4/6, PI3K, and BRD4. SRX3177 displays excellent kinome and BET bromodomain selectivity and marked cytotoxicity across multiple tumor types. Our results demonstrate that SRX3177 is efficacious and non-toxic to normal cells in vitro in a wide range of tumor models suggesting further evaluation of this distinct chemotype in resistant cancer models.

\section{Acknowledgements}

We thank Brianna Klein and Forest Andrews for helping with experiments. This work was supported by the NIH grants CA192656 (D.L.D.), FD005113 (D.L.D., A.M.B.), GM135671 (T.G.K.), GM125195 (T.G.K.) and the Tower Cancer Research Foundation grant (A.M.B.). K.R.V. is a CFReT Fellow.

\footnotetext{
Author details

'Division of Hematology-Oncology, Department of Medicine, Moores Cancer Center, University of California San Diego, La Jolla, CA, USA. ${ }^{2}$ Department of
} 
Pharmacology, University of Colorado School of Medicine, Aurora, CO, USA ${ }^{3}$ Division of Pediatric Hematology and Oncology, Department of Pediatrics, Moores Cancer Center, University of California San Diego, La Jolla, CA, USA. ${ }^{4}$ SignalRx Pharmaceuticals, San Diego, CA, USA. ${ }^{5}$ Department of Cell Biology, Instituto de Biomedicina de Sevilla, Universidad de Sevilla, Sevilla, Spain

\section{Author contributions}

A.M.B., K.R.V., S.J., G.A.M., F.M.V., A.S., and D.P. performed experiments and together with A.B.M., T.G.K., and D.L.D. analyzed the data. A.M.B., T.G.K., and D.L.D. wrote the paper with input from all authors.

\section{Data availability}

The atomic coordinates and structure factors have been deposited in the Protein Data Bank under accession code 6WW8. All other relevant data supporting the key findings of this study are available within the article and its Supplementary information files or from the corresponding authors upon reasonable request.

\section{Conflict of interest}

D.L.D. and G.A.M. acknowledge financial interest in SignalRx

Pharmaceuticals, Inc

\section{Publisher's note}

Springer Nature remains neutral with regard to jurisdictional claims in published maps and institutional affiliations.

Supplementary Information accompanies the paper at (https://doi.org/ 10.1038/s41421-020-0181-z).

Received: 31 March 2020 Accepted: 9 May 2020

Published online: 28 July 2020

\section{References}

1. Dey, N., Leyland-Jones, B. \& De, P. MYC-xing it up with PIK3CA mutation and resistance to PI3K inhibitors: summit of two giants in breast cancers. Am. J. Cancer Res. 5, 1-19 (2015).

2. Bendell, J. C. et al. Phase I, dose-escalation study of BKM120, an oral pan-Class I PI3K inhibitor, in patients with advanced solid tumors. J. Clin. Oncol. 30 282-290 (2012).

3. Janku, F. et al. PI3K/AKT/mTOR inhibitors in patients with breast and gynecologic malignancies harboring PIK3CA mutations. J. Clin. Oncol. 30, 777-782 (2012).

4. Stratikopoulos, E. E. et al. Kinase and BET Inhibitors Together Clamp Inhibition of PI3K Signaling and Overcome Resistance to Therapy. Cancer Cell 27, 837-851 (2015).

5. Matulonis, U. A. et al. Phase I dose escalation study of the PI3kinase pathway inhibitor BKM120 and the oral poly (ADP ribose) polymerase (PARP) inhibitor olaparib for the treatment of high grade serous ovarian and breast cancer. Ann. Oncol. 28, 512-518 (2016).

6. Morales, G. A. et al. Synthesis and cancer stem cell-based activity of substituted 5-morpholino-7H-thieno [3, 2-b] pyran-7-ones designed as next generation PI3K inhibitors. J. Med. Chem. 56, 1922-1939 (2013).

7. Andrews, F. H. et al. Dual-activity PI3K-BRD4 inhibitor for the orthogonal inhibition of MYC to block tumor growth and metastasis. Proc. Natl. Acad. Sci. USA 114, E1072-E1080 (2017).

8. Carlino, L. \& Rastelli, G. Dual kinase-bromodomain inhibitors in anticancer drug discovery: a structural and pharmacological perspective. J. Med. Chem. 59 9305-9320 (2016).

9. Divakar, S. K. et al. Dual inhibition of CDK4/Rb and PI3KAKT/mTOR pathways by ON123300 induces synthetic lethality in mantle cell lymphomas. Leukemia 30, 86-93 (2016).

10. Filippakopoulos, P. et al. Selective inhibition of BET bromodomains. Nature 468, 1067-1073 (2010). 